\title{
Breakdown voltage in multi walled Carbon- nanotubes during low voltage (4V) DC switching
}

\author{
J.W.McBride \\ Dept of Mechanical Engineering \\ University of Southampton Southampton, UK \\ jwm@soton.ac.uk
}

\author{
T. G. Bull \\ Dept of Mechanical Engineering \\ University of Southampton Southampton, UK \\ tb10g11@soton.ac.uk
}

\begin{abstract}
A metallic coated carbon nanotube surface, developed for use in a micro-electromechanical (MEMS) switching device, is investigated for performance, when switching 4 VDC, with current levels between 10 to $600 \mathrm{~mA}$; with the objective of understanding the upper limits of the surface performance. The surface used is a Gold Coated (500 $\mathrm{nm})$, forest of multi-walled carbon nanotubes $(50 \mathrm{\mu m})$, referred to as $\mathrm{Au} / \mathrm{CNT}$. The surface has been previously investigated for endurance at low current levels of $4 \mu \mathrm{A}$, to characterize the cold switching performance over 4 billion switching cycles and tested in a cantilever MEMs test system with $10 \mathrm{~mA}$ (hot switching) over 0.5 billion cycles. This investigation studies the current loading level with a focus on the transient voltages during the opening process. The investigation is undertaken using a piezoelectric (PZT) actuator, as part of an in-situ test apparatus (ICE). Results are compared to the transient voltage on a reference carbon nanotube surface without the gold coating, under the same switching conditions. The results for the $\mathrm{Au} / \mathrm{CNT}$ surface show that the voltage transients are dominated by molten metal bridge phenomena up to $300 \mathrm{~mA}$ above which a previously unreported switching transient is observed. The higher current levels ( 300 to $600 \mathrm{~mA}$ ) are shown to puncture the gold surface after 10's of switching cycles exposing the CNT. Under this condition the exposed CNT become conductive and thermally decompose (pyrolyze).
\end{abstract}

Keywords-MEMS switching, Multi-walled Carbon Nanotubes, current loading limits.

\section{INTRODUCTION}

For more than 30 years the promise of a commercial MEMS relay switching device has been on the horizon. MEMS fabrication technology opening the door to a switching device which combines the attributes of mechanical switching, with microfabrication technologies, for mass produced micro devices. Today these promises are made in the context of $5 \mathrm{G}$ switching systems, $[1,2,3]$, but to date the application has not fully materialised; because electrical contact switching surfaces are prone to two fundamental mechanism of failure; adhesion at very low force (with precious metals) and surface wear. The combination of the two failure mechanisms often results in failure of devices after a few thousand switching cycles, while the ambition is to switch billions of cycles. Whilst MEMS relays boast a number of advantages over PIN and FET devices, notably the high isolation, low on-resistance and excellent frequency performance; one of the major disadvantages of MEMS relays is that the electric contacts are prone to failure $[3,4]$. The surface wear phenomena is a complex interaction of physical and chemical processes, here described in four broad categories linked to the nature of the circuit. The four categories are described in the context of a Au/CNT surface outlined below and assume that there is no electrical breakdown between the opening contacts, normally associated with the onset of arcing or other forms of electrical discharge.

1. Cold switching (dc). In this condition the electrical contacts are exposed to mechanical cycling only and lifetime may reach billions of cycles. The failure mode is linked to adhesion. In a recent study, [5], with a very small current (6 $\mu \mathrm{A})$, a surface was tested to 4 Billion switching cycles without failure.

2. Hot switching (dc), (MMB mode). As the contacts separate the voltage drop across the interface will exceed the softening, melting and boiling voltage of the metallic surface, leading to the formation of a molten metal bridge (MMB) which will disrupt the surface, [6]. Fig.1 shows a schematic of a MMB and the associated transfer process. It has been shown that the transfer of material between the surfaces is linked to the bulk thermal properties of the surfaces, [7].

3. Hot switching (dc), (DL mode). In addition to the MMB linked fine transfer of material, it has be shown that for surfaces with coatings; e.g. the Au coated forest of multiwalled carbon nanotubes referred to as Au/CNT; that in addition to the MMB event there are delamination (DL) events linked to adhesion. An investigation has shown that DL events occur as frequently as $30 \%$ of all switching operations, on the Au/CNT surface, [8]. The delamination event occurs when the surfaces adhere at closure and are forced apart by an actuator.

4. Hot and Cold switching (RF). In the RF application, power loss in the transmitted signal (insertion loss) is low due to the minimal on-state impedance of a metal-metal interface, while the mechanical opening mechanism provides an 'air gap' in the off-state, giving excellent signal attenuation and a large ratio in the off-to-on impedance (isolation) [1,2]. A recent investigation using a full MEMS device, with RF hot switching of a Au-CNT composite contact registered a limited life-time of a few thousand cycles, [9]; an improvement over the metal-to-metal interface by a factor of 3 . There are few studies comparing the DC and RF performance of MEMS devices; but in a related micro relay study, a good performance with RF loads up to $37 \mathrm{~W}$ at frequencies up to $3 \mathrm{GHz}$, was noted, [10].

In summary contact failure mechanisms at low voltage $(<12 \mathrm{~V})$ and low current $(<1 \mathrm{~A})$ conditions, be can be classified as either; adhesion, where the contacts stick; or by an excessive increase in contact resistance, due to surface wear [11,12]. Gold (Au) provides excellent conductive properties and protection against corrosion; however, these properties are offset by the relative softness, vulnerability to wear, and self-adhesion [13,14]. The use of harder metallic surfaces with higher melting points, such as Tungsten or 
Ruthenium can reduce contact wear [3,4]; but have lower conductivity and thus a higher contact resistance.

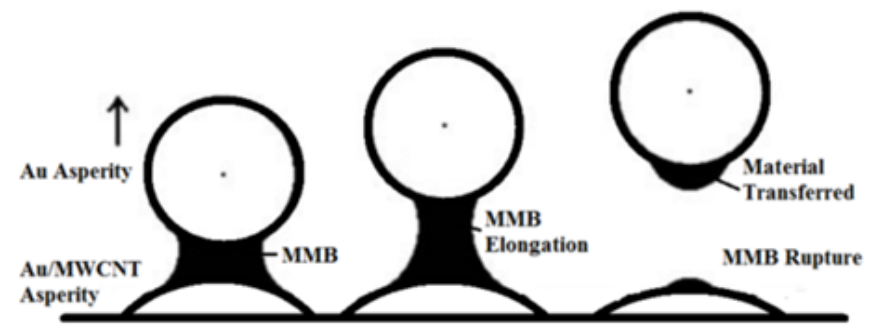

Fig. 1. The fine transfer model showing the molten metal bridge on a $\mathrm{Au} / \mathrm{CNT}$ surface as the contacts open, [5,17].

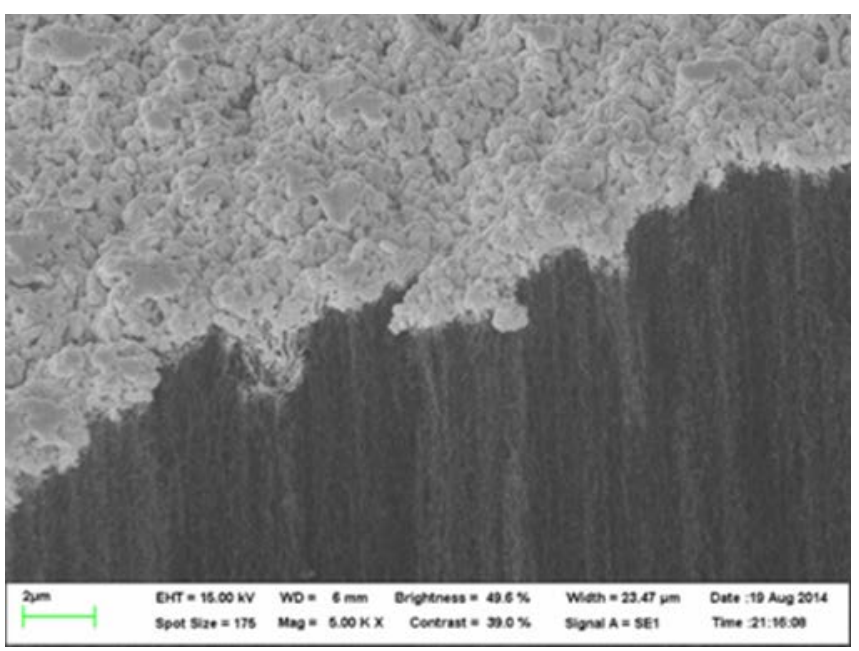

Fig. 2. Au/CNT surface showing the Au coating at the edge of the sample, with Au penetration into the CNT surface.

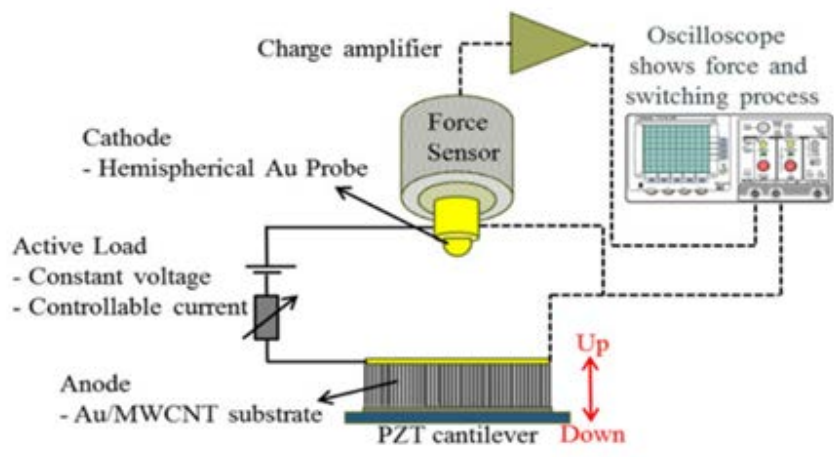

Fig. 3. Schematic of the test circuit with the force sensor and actuator.

As noted above a solution to the durability issue has been developed using a composite contact material, consisting of compliant sub-layer of vertically aligned multi-walled carbon nano-tubes (MWCNT) with a coating of Au, generally referred to as $\mathrm{Au} / \mathrm{MWCNT}$ or simply Au/CNT (in this paper), shown in Fig.2 and Fig.3. This surface has been shown to provide both low contact resistance $(<1 \Omega)$ and enhanced tolerance for wear, [11, 15-17]. Under the "hot switching" condition with a DC power supply of $4 \mathrm{~V}$ and $10 \mathrm{~mA}$, a MEMS test device was tested to 0.5 Billion operations without failure in [17]. The material is yet to be tested in a full MEMS device but a similar composite material using non-aligned nanotubes has been investigated in a MEMS device in [9], where the benefits to the durability were not significant, as noted above. This suggests that the alignment of the carbon nanotubes is very important for the improved performance.
Using the vertically aligned CNT structure the composite deforms, allowing the contacts to conform to each other, increasing the area available for conduction [16].

In this study, the upper current loading limits of the $\mathrm{Au} / \mathrm{CNT}$ surface are explored. In [8], using the same experimental approach, an increase in the supply voltage to $8 \mathrm{~V}$ was investigated. It was shown that an increase in the supply voltage resulted in an increase in the wear rate of the surfaces. It should be noted, that the MMB transfer processes is thought to be only linked to the duration of the MMB event and not to the supply voltage. This unexpected result has not been fully explored.

This work focuses on the dc current load level to a maximum of $600 \mathrm{~mA}$. At these high current levels, the upper layer of $\mathrm{Au}$ can be expected to be disrupted exposing the underlying CNT layer.

\section{MethodOLOGY}

\section{A. In-situ Contact Evolution (ICE) apparatus}

In this experiment the practical upper limit to the hot switched current carrying capacity of Au/CNT is investigated. $\mathrm{An} \mathrm{Au} / \mathrm{CNT}$ contact is paired with an Au coated hemisphere contact and switched at 4 VDC while the load current is varied. The power supply used is a Sonnenschein A504 lead acid battery, with a terminal voltage that will hold above 3.5 $\mathrm{V}$ for a discharges of 49 A for up to 3 minutes. The listed (fully charged) internal resistance is $48 \mathrm{~m} \Omega$.

The ICE testing platform, $[8,18]$, utilises a commercial metrology instrument (XYRIS 2020), TaiCaan Technologies Ltd, [19]; integrating a 3D laser profiling system into the testing platform such that after a set number of cycles the contact surfaces can be separated and measured before returning the surfaces to the identical switching position to allow the switching tests to continue. The ICE apparatus is mounted on anti-vibration table in a temperature controlled environment $22 \pm 0.2{ }^{\circ} \mathrm{C}$. The contact resistance $\left(R_{c}\right)$ is measured using the 4-wire method, using a Keithley microohm meter 580, allowing the contacts to settle over a 30 second period

\section{B. Contact Materials}

The hemisphere contact shown in Fig. 3 is a 2 mm diameter stainless steel ball, sputter coated with a $20 \mathrm{~nm}$ layer of $\mathrm{Cr}$ to enhance adhesion of the $500 \mathrm{~nm}$ Au layer, [15]. The planar contact surface is a Au/CNT composite, shown in Fig.2. The contact is manufactured on a $\mathrm{SiO}_{2}$ on $\mathrm{Si}$ substrate that is sputter coated with a layer of $\mathrm{Al}_{2} \mathrm{O}_{3}$ followed by an Fe catalyst layer. Carbon nanotubes are grown in a thermal reactor to 50 $\mu \mathrm{m}$ height and sputter coated with a $500 \mathrm{~nm}$ Au layer, (referred to as a 505 surface). The Au penetrates into the top of the nanotube forest resulting in a metal-matrix-composite material. The composite provides an upper layer with conductive properties and contact resistance properties close to that of a Au thin-film coating. The vertically aligned carbon nanotubes provide a compliant sublayer allowing the composite to conform, increasing contact area, [20].

\section{Experimental Conditions}

The PZT actuator is driven by a $0.5 \mathrm{~Hz}$ square wave, with a steady state closure force of $150 \mu \mathrm{N}$. For each experiment, the contact is cycled 100 times with no current to allow the 
contact interface to stabilize or "bed in". The contacts are tested with a 4 VDC supply. The contact opening transient voltages are captured for fifty consecutive opening events, at nominal current values of 10, 20, 50, 100, 200, 350, 450 and $600 \mathrm{~mA}$. The test circuit is arranged as per Fig 3 . The 4 -wire contact resistance is recorded before and after each experiment.

Two conditions are investigated;

1. Au/CNT. The Au/CNT (505) material as detailed, is mated with the Au coated ball as shown in Fig .3.

2. CNT only. A $50 \mu \mathrm{m}$ high CNT surface is mated with the Au coated ball (see Fig.10).

\section{RESULTS}

\section{A. Experimental Conditon 1:Contact Resistance.}

The contact resistance before and after 50 hot switched cycles against the closed circuit (switching) current is presented in Fig. 4, for the Au/CNT surface. The initial, contact resistance $(R c)$ is shown to be $440 \mathrm{~m} \Omega$ for all conditions. The figure shows that after 50 hot switching cycles there is a slight decrease in the contact resistance for switching currents between 10 and $300 \mathrm{~mA}$. As the current is increased to 345, 450 and $600 \mathrm{~mA}$ the contact resistance after switching increases by 48,122 and $430 \mathrm{~m} \Omega$ respectively. The increase in contact resistance after the higher current switching cycles is linked to the removal of the Au coating from the CNT surface, as detailed in the next section.

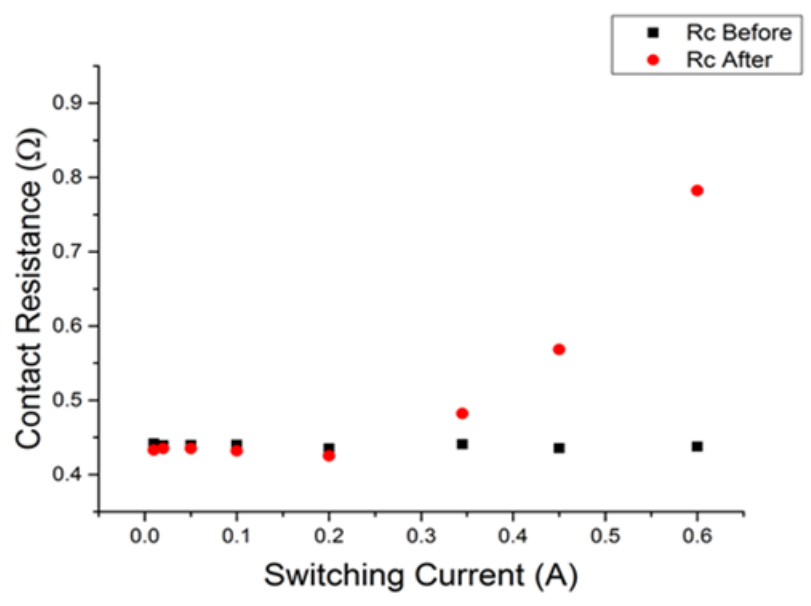

Fig. 4 The change in contact resistance (Rc) after 50 hot switching cycles as current is varied.

\section{B. Experimental Conditon 1: Voltage Transients}

The contact opening voltage transients are synchronized by defining $\mathrm{t}=0$ as the point where the contact potential reaches 3 V. Fig.5 shows the average waveforms over 50 cycles, for each current.

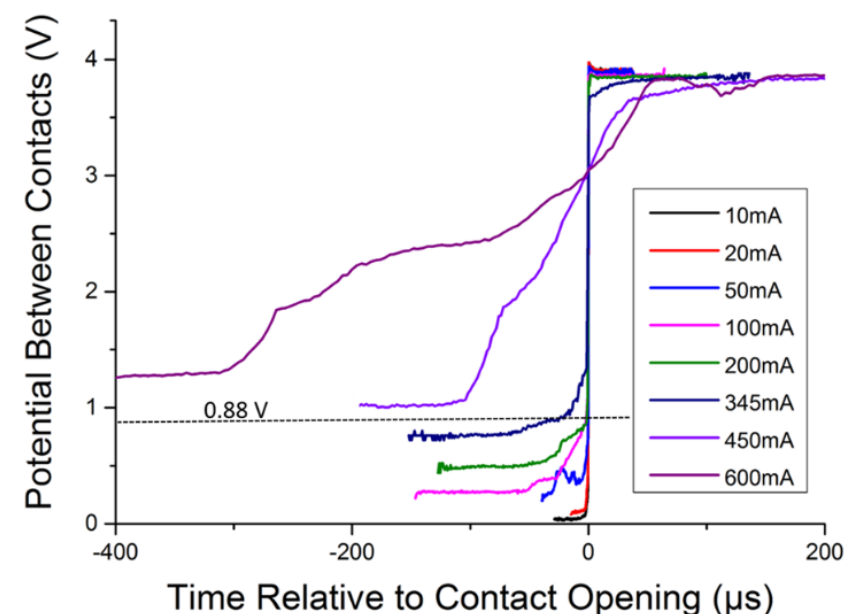

Fig. 5 Average voltage transients 4 VDC, 10 to $600 \mathrm{~mA}$ with the boiling voltage for Au indicated by horizontal dashed line

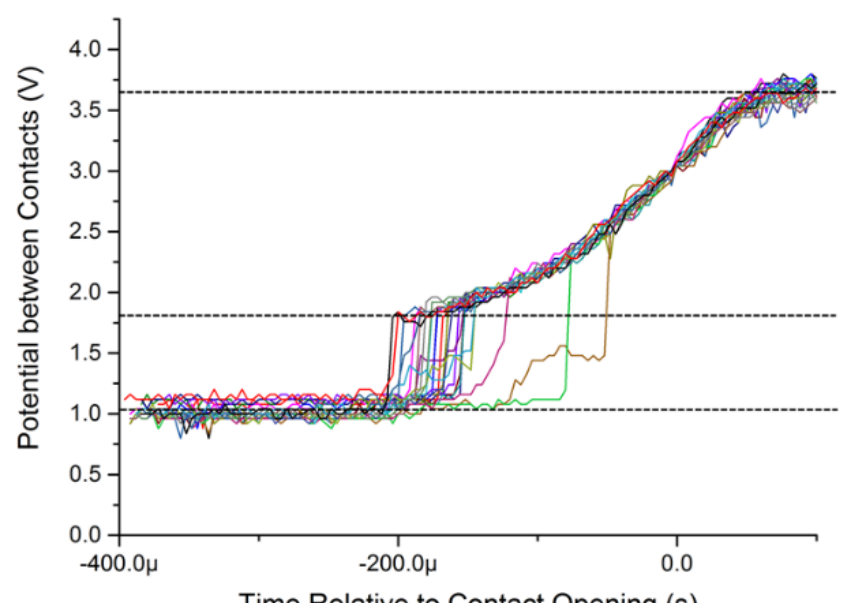

Fig. $6 \mathrm{MMB}$ voltage transients over 25 consecutive opening events with at $450 \mathrm{~mA}$ circuit current

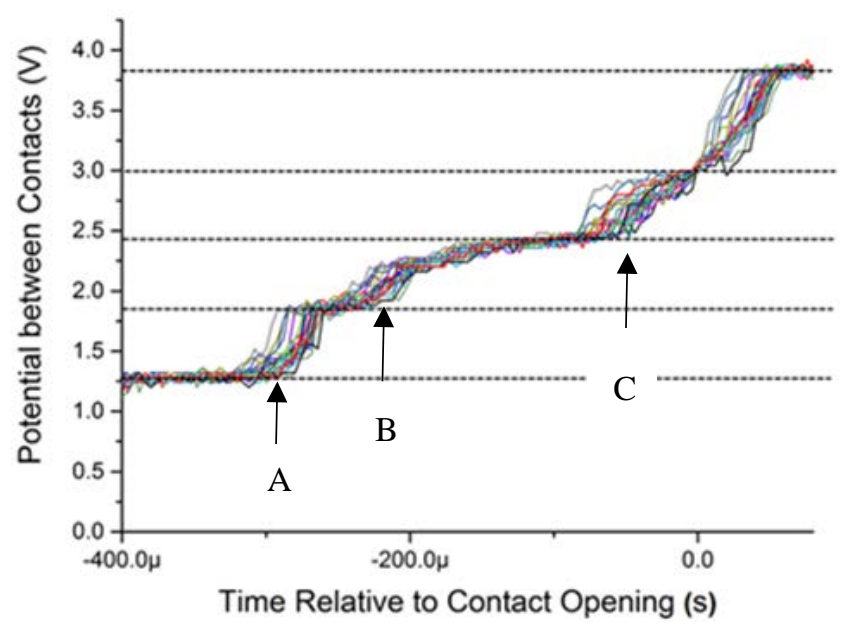

Fig. 7. The contact potential during 25 consecutive opening events with at $600 \mathrm{~mA}$ circuit current

In Fig 5, the closed contact voltage-drop across the $\mathrm{Au} / \mathrm{CNT}$ and ball contact is initially level and stable. The closed circuit voltage-drop $\left(U_{R}\right)$, prior to the opening event is measured across the sum of the bulk resistance and the contact resistance $(R c)$ of the interface and is approximately $5 \Omega$. From the contact resistance values in Fig.4, this implies that most of the voltage drop is across the bulk resistance, composing of the surface of the $\mathrm{Au} / \mathrm{CNT}$ and the 
interconnects. As the steady state current is increased the closed circuit voltage-drop across the interface increases; at $600 \mathrm{~mA}, U_{R}$ is $1.25 \mathrm{~V}$. At the start of the opening process, for current values between 10 and $200 \mathrm{~mA}$, there is a gradual rise in voltage linked to the MMB phenomena $\left(U_{M M B}\right)$ of softening, melting $(0.4 \mathrm{~V})$ and boiling $(0.88 \mathrm{~V})$ of the $\mathrm{Au}$ surface. Not all of the voltage transients reach the $0.88 \mathrm{~V}$ level, as indicated by the average waveforms. The time between the onset of the voltage increasing and the contact opening (MMB duration) increases linearly with current, as shown in [5,17]. The $0.88 \mathrm{~V}$ level indicated in the figure is shown relative to zero, but the voltage associated with the MMB $\left(U_{M M B}\right)$ is an additional voltage-drop; such that the measured transient voltage $(v)$, prior to the open circuit, is;

$$
v=U_{R}+U_{M M B}
$$

We refer to this type of voltage transient in the closed circuit current range $0-300 \mathrm{~mA}$ as Type 1 . For the higher nominal current values of 450 and $600 \mathrm{~mA}$, in Fig. 5, there is a noticeable change in the voltage transient across the opening contacts. We refer to this as Type 2. The Type 2 transients are further explored in Fig. 6 and 7, where the individual waveforms of the opening voltage transients are shown over 25 consecutive cycles. In Fig. 6 for $450 \mathrm{~mA}$, the data shows a consistent step of approximately $0.8 \mathrm{~V}$ as the contacts open. The step does not occur at the same time relative to the open circuit. After the step there is a further observed phenomenon characteristic of this Type 2 transient, shown as a gradual increase in voltage $\left(U_{c}\right)$ to the open circuit voltage (4V). For the new phenomena, we should adjust Eq (1) as follows;

$$
v^{\prime}=U_{R}+U_{C}
$$

Fig 7, for the maximum nominal current value of 600 $\mathrm{mA}$, shows a further complexity to the Type 2 voltage transient with 3 or 4 steps observed (A,B,C noted in Fig.7), where there is a distinct beginning to the opening event characterized by sudden increase in contact potential from 1.25 to $1.8 \mathrm{~V}$ (at A). The resultant first step voltage of $1.8 \mathrm{~V}$ is also shown in Fig. 6 for the lower current of $450 \mathrm{~mA}$, but in Fig 7 there are two additional steps; the second step at B to $2.4 \mathrm{~V}$ and the third at $\mathrm{C}$ to $3.0 \mathrm{~V}$. The observed first steps in the opening transient voltage for the $450 \mathrm{~mA}$ and $600 \mathrm{~mA}$ are approximately $0.8 \mathrm{~V}$ and $0.6 \mathrm{~V}$ respectively.

\section{Experimental Conditon 1, Contact Surface.}

The location of the $600 \mathrm{~mA}$ switching site is shown Fig.8 with a surface height map of the same region in Fig. 9. The dark regions in Fig. 8, are areas where the Au has been removed. In Fig.9 the same regions are shown in black corresponding to regions where the laser system (XYRIS 2020) has a near zero return when reflecting from a carbon surface. In Fig.9 the black regions are at high surface (red) locations showing that Au removal has occurred at asperity tips, exposing the underlying CNT. Four enclosed regions are highlighted in both Fig's. Sites A and B show two lower and flatter regions, while sites $\mathrm{C}$ and $\mathrm{D}$ indicate the location of two raised regions. To verify, the dark regions were exposed to energy dispersive $\mathrm{x}$-day (EDX) analysis and show an increase carbon content in the darker regions.

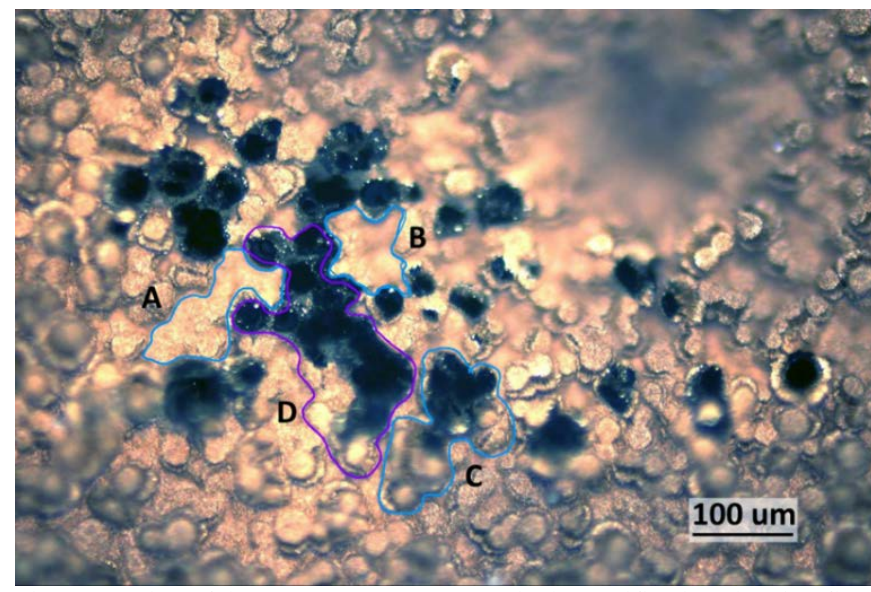

Fig. 8 Location of the $600 \mathrm{~mA}$ test at 200x optical magnification showing flat regions (A and $\mathrm{B}$ ) and asperities with damage (C and D)

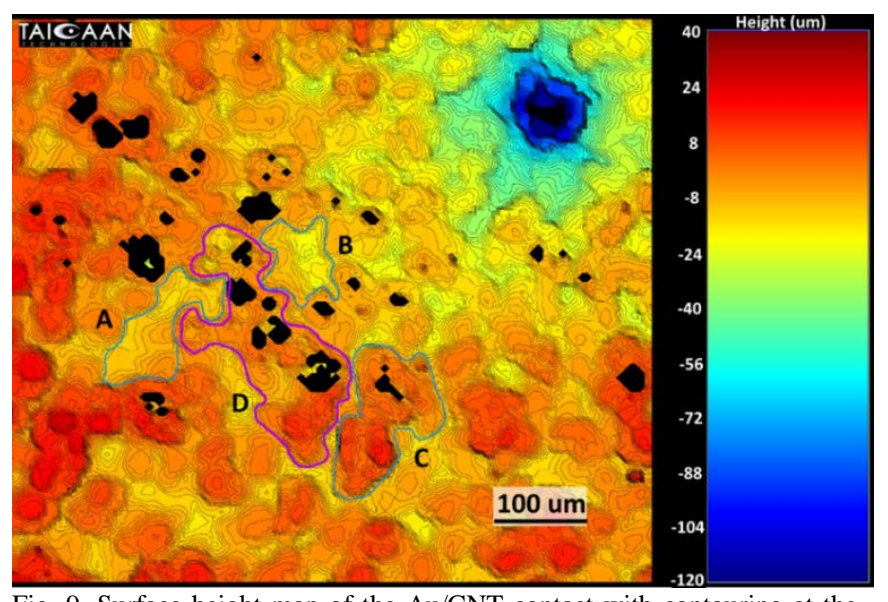

Fig. 9. Surface height map of the $\mathrm{Au} / \mathrm{CNT}$ contact with contouring at the same location as Fig. 8. Regions A and B are lower plateaus. Regions C and $\mathrm{D}$ are raised asperities.

\section{Experimental conditon 2, Voltage transients with CNT surface.}

This experiment is undertaken to investigate the Type 2 transients noted in the previous section. In this experiment the planar contact is vertically aligned CNTs. The experimental arrangement is shown in Fig.10, and shows the Au coated ball in contact with the CNT surface. The CNT bulk resistance and contact resistance is significantly higher than the Au coated surface $(\sim 28 \Omega)$, limiting the maximum circuit current to 100 $\mathrm{mA}$. Fig. 11 shows the result of 10 consecutive opening waveforms observed with 4 VDC and $20 \mathrm{~mA}$.

The higher bulk resistance combined with an higher contact resistance, results in an increased voltage drop, $\left(U_{R}\right)$, when compared to the Au coated surface, $1 \mathrm{~V}$ and $0.02 \mathrm{~V}$ respectively, at $20 \mathrm{~mA}$. The voltage transients are similar to those observed in experiment 1 , at $600 \mathrm{~mA}$, with a gradual increase in voltage to a peak of approximately $3 \mathrm{~V}$ prior to the open circuit voltage, of $4.2 \mathrm{~V}$; reflecting the relationship Eq. (2). It is also noted that the duration of the voltage transients are significantly longer (x1000) than in experiment 1 . In this experiment the CNT surface will be more elastic and with a lower stiffness, than the Au/CNT surface. The ball contact will therefore penetrate further into the CNT, taking correspondingly longer for the contacts to open. In this case, the physical process leading to the voltage $\left(U_{C}\right)$ is not associated with the MMB phenomena between opening metallic contacts. 


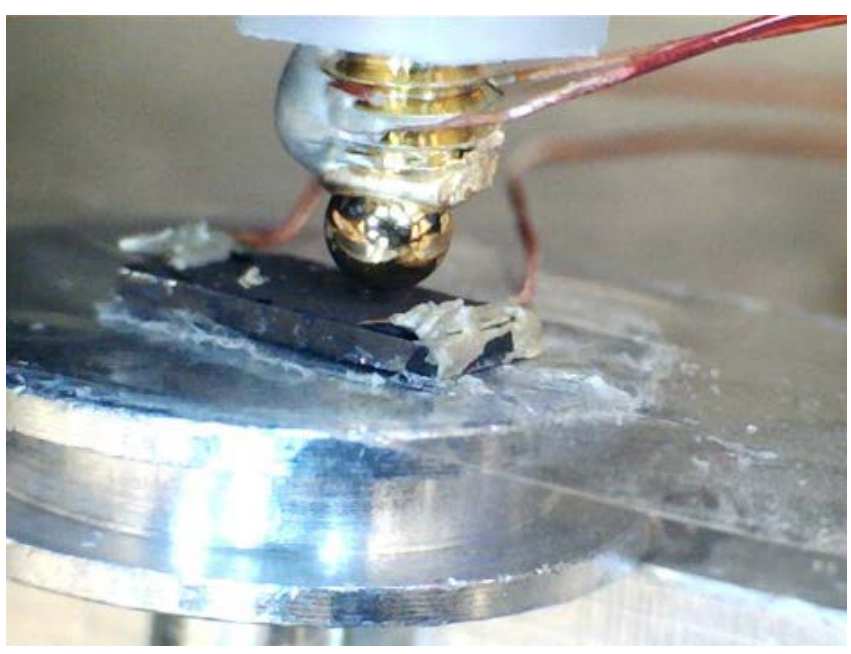

Fig.10 The arrangement of upper Au coated sphere contact and lower vertically aligned CNT lower contact.

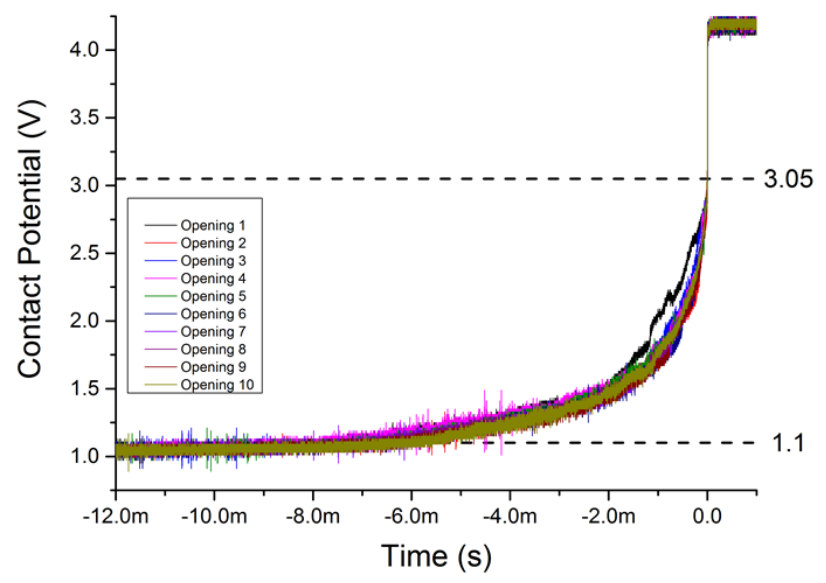

Fig. 11 Ten voltage transients on pure CNT, at $4 \mathrm{~V} 20 \mathrm{~mA}$

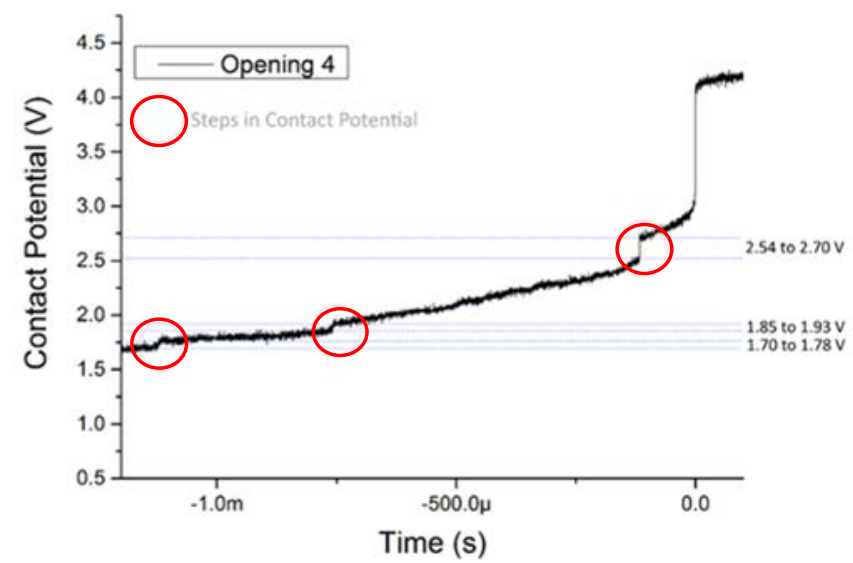

Fig. 12 Detail of a single voltage transient on CNT, at $4 \mathrm{~V} 20 \mathrm{~mA}$ with steps in the contact potential indicated by a red circle

To investigate the phenomena further, Fig 12, shows a single opening event (4th) taken from the data in Fig 11, with detail $1.2 \mathrm{~ms}$ before the contact opens. Three steps in the contact potential are apparent, as indicated by a ring while the corresponding voltage levels are shown. At $1100 \mu$ s before the contact opens the first step in contact voltage drop increases from 1.70 to $1.78 \mathrm{~V}(0.08 \mathrm{~V})$. At $750 \mu \mathrm{s}$ before contact opening there is a second step from $1.85 \mathrm{~V}$ to $1.93 \mathrm{~V}(0.08 \mathrm{~V})$. At $100 \mu$ s before the contact opens, there is a third step from
2.54 to $2.70 \mathrm{~V}(0.16 \mathrm{~V})$. The steps appear in all opening waveforms with the CNT only contact.

\section{DISCUSSION}

The voltage transients shown during the opening events below $350 \mathrm{~mA}$ are all associated with the MMB process, showing the characteristics of softening, melting $(0.4 \mathrm{~V})$ and boiling $(0.88 \mathrm{~V})$ of the Au surfaces, referred to here as Type 1. For voltage transients above $350 \mathrm{~mA}$, there are new observations referred to as a Type 2 opening.

\section{A. Experimental Condtion 1. Type 2 (350-600 mA data)}

There are 3 stages in the Type 2 opening voltage transients. Stage 1 , occurs at the start with a steady state current flow through the contact interface, leading to a steady state voltage $U_{R}$. Stage 2 occurs at the onset of opening, while stage 3, occurs after the first opening step and is a new undefined characteristic voltage in Eq. (2).

The stage 3 process as observed in the data in Fig's 4, 8 \& 9, and must be linked to a conduction process through a CNT surface only. This is evidenced in the resistance in Fig 4, which shows that after high current (350-600 mA) cycling that the contact resistance has increase by a factor between 50 $100 \%$. Indicating the surface changes observed in Fig 8 \& 9 where there is clear evidence of the exposed CNT surface. When the contact is closed it is likely that some conduction will occur between the Au ball and the remaining Au on the $\mathrm{Au} / \mathrm{CNT}$ surface. This suggests that the initial opening, stage 2 , is very likely to show the regular MMB phenomena, where the step corresponds to a melting and or boiling of the surface. In Fig. $6(450 \mathrm{~mA})$ the step is consistently $0.8 \mathrm{~V}$ while in Fig $7(600 \mathrm{~mA})$ the first step at A in the Fig is approximately 0,6 $\mathrm{V}$. It is interesting to note that the resultant step voltage (1.8 $\mathrm{V}$ ) is the same in both 450 and $600 \mathrm{~mA}$ examples. The Stage 3 process after the first step is characterised by a continuous increase in the transient voltage to the open circuit value. It is proposed that this third stage characteristic is representative of a CNT only contact.

\section{B. Experiment 2. CNT only surface, $20 \mathrm{~mA}$}

The voltage transient in Fig.11 is considered to reflect a gradual reduction in the area of contact between the CNT surface and the Au coated hemisphere contact; this is shown schematically in Fig. 13. As the balled contact moves away from the lower CNT surface the number of individual CNT fibres in contact with the ball reduces, to the point where only be a few CNTs are in contact. The reduction in conduction paths will lead to the trend in Fig. 11. This is an interface resistance and not the contact resistance normally associated with bulk materials, [21]. The interface resistance will consist of a number of individual nanotubes in contact with the Au ball, with each nano tube conducting; as the contact separate fewer and fewer CNT will be in the conduction path. This must eventually result in a single nanotube in contact with the ball, as shown at position 3 in Fig. 13. 


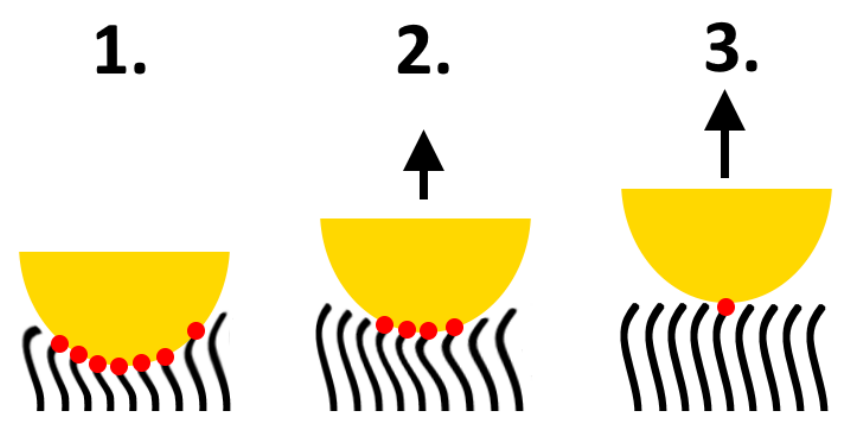

Fig. 13 A diagram of showing the number of CNT is contact with the Au coated ball as the contacts are 1) closed, 2) opening, and 3) only a single nanotube still in contact. The red dots highlight the fibres in contact.

The data in Fig 12, shows a single opening event from 1 ms prior to the open circuit. As fewer and fewer CNTs make contact the current flow in the remaining contacting fibres will increase resulting in the sublimation, (pyrolyze) of the multi-walled CNTs. Experimental evidence [22-24] of single current carrying multi-walled CNTs have shown a series of voltage steps associated with a thinning of the CNT. The steps were linked to the consecutive thermal failure of the nanotube walls by current overload in both vacuum and ambient conditions [24]. In [24], the bias voltage at which the breakdown occurs is between 2.5 and $5.2 \mathrm{~V}$ in air. The paper also notes that in the case of bundles of CNT (the case in this paper) that the resulting transients were complex with multiple events. An example of the thermal (pyrolyze effect) process in illustrated in Fig. 14, where the outer walls of the MWCNT, thermally fails (pyrolyzes) corresponding to step changes in the resistance. Thus, the steps in Fig.12, could be explained by a combination of the discrete nature of the surface were fewer and fewer CNTs conduct combined with the thermal process when only one or two remaining CNTs are in contacts.

In a further observation, in [22], wall ruptures were observed to occur in the middle of the CNT rather than at the contact site; as shown in Fig 14. It has been observed previously [11], that the Au/CNT planar surface can transfer to the Au ball surface with the upper 5 to $6 \mu \mathrm{m}$ of the surface sticking to the ball contact, as shown in Fig 15.
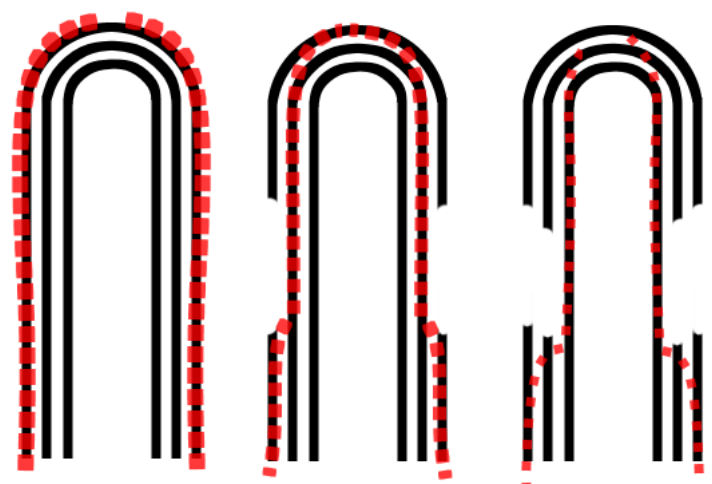

Fig. 14 As the current overloads the CNT wall, the wall thermally fails (pyrolyzes) and a step change in resistance of the current path is observed.

\section{CNT to CNT contact}

For the experimental condition investigated it is possible that the upper planar Au/CNT surface transfers to the ball contacts. Fig.15, is a microscope image of the hemispherical ball after testing 140 Million cycles at $50 \mathrm{~mA}$, from experiments in [5]; where the darkened areas are CNT fibres which have been removed from the Au/CNT surface. In the image the CNT surface is shown covering an area of approximately $0.5 \times 0.5 \mathrm{~mm}$. It has been shown in surface analysis [5,8] that when the lower Au/CNT surface delaminates, that the CNT break at a length of typically $5 \mu \mathrm{m}$ from the top of the Au/CNT surface or a $50 \mu \mathrm{m}$ growth of CNT.

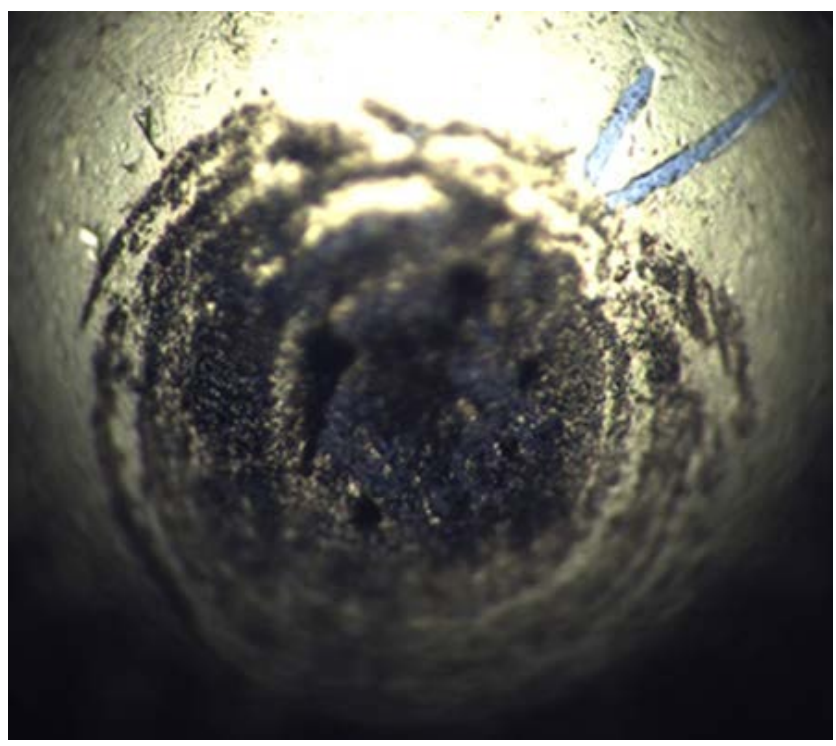

Fig. 15. Au balled contact after 4V 50mA 140M cycles, [5], (FOV) 0.7 x $0.6 \mathrm{~mm}$. Dark areas are CNT transferred from the Au/CNT surface.

During the higher current switching it is evident that some of the Au surface in Experiment 1, has transferred to the ball contact, as evidenced by the loss of Au from the lower $\mathrm{Au} / \mathrm{CNT}$ surface in Fig's 8 \& 9. Previous investigations have shown, as evidenced in Fig 15, that when this happens some of the upper CNT surface is also removed and adheres to the ball contact. There are 3 possible outcomes;

1. Planar surface has exposed CNT in contact with the Au ball.

2. Planar Surface has the Au upper surface in contact with CNT transferred to the ball.

3. Planar Surface has exposed CNT in contact with CNT transferred to the ball. This would be a CNT to CNT contact.

If we assume that individual CNT fibres are in contact across the interface then as the contact separate in Experiment 1 , we could expect to see a combination of the two process; firstly a reduction in current conduction paths as shown in Fig. 11, coupled with the second process of pyrolyze shown in Fig. 12. There is evidence that at the higher current level of 600 $\mathrm{mA}$ in Fig. 7, that both processes could be active.

\section{CONCLUSION}

$\mathrm{Au} / \mathrm{CNT}$ contacts are hot switched at currents between 10 and $600 \mathrm{~mA}$. Above $350 \mathrm{~mA}$ the Au/CNT shows early signs of wear and the transient opening voltage changes from a typical MMB (Type 1) to a new phenomenon (Type 2). Under these conditions the Au film is shown to have been punctured after 10's of switching cycles, exposing the subsurface CNT and allowing CNT to contact with the opposing Au coated ball. The mechanism for the newly observed, Type 2 contact 
opening waveforms is linked to a few remaining CNT's carrying the current pathway during the final moments of contact separation. The voltage step observed are linked to the discrete nature of the surface combined with a thermal process where the CNT shells sublime, (pyrolyze effect).

The results show that the Au/CNT surface has a upper current level limited to the Type 1 , MMB type wear process. Above this Type 2, the surface is disrupted exposing the lower CNT surface, resulting in a higher contact resistance.

\section{ACKNOWLEDGMENT}

The authors would like to acknowledge the software developed by Dr K. Cross at TaiCaan Technologies Ltd.

\section{REFERENCES}

[1] G. M. Rebeiz, RF MEMS: theory, design, and technology: John Wiley \& Sons, 2004.

[2] Jacapo Iannacci, RF-MEMS Technology for High performance passice, IOP Books, 2017.

[3] B. F. Toler, R. A. Coutu, and J. W. McBride, "A review of microcontact physics for microelectromechanical systems (MEMS) metal contact switches," Journal of Micromechanics and Microengineering, vol. 23, no. 10, Oct, 2013.

[4] A. Basu, G. Adams, and N. McGruer, "A review of micro-contact physics, materials, and failure mechanisms in direct-contact RF MEMS switches," Journal of Micromechanics and Microengineering, 2016, vol. 26, no. 10, pp. 104004.

[5] JW McBride, TG Bull, "The in-situ wear of a hot and cold switched $\mathrm{Au}$ on $\mathrm{Au}$ coated MWCNT electrical contact for a MEMS switch application”, 2019 IEEE Holm Conference on Electrical Contacts, pp 149-155.

[6] J. McBride, "The wear processes of gold coated multi-walled carbon nanotube surfaces used as electrical contacts for microelectro-mechanical switching,” Nanoscience and Nanotechnology Letters, 2010, vol. 2, no. 4, pp. 357-361.

[7] M. Down, "Mechanical and Electrical Characterisation of Carbon Nanotubes Composite Surfaces for MEMS switching applications” Ph.D thesis, University of Southampton UK, 2015.

[8] T.G. Bull, J. W. McBride, and L. Jiang. "The influence of Circuit Parameters on Molten Bridge Surface Degradation in a $\mathrm{Au} / \mathrm{MWCNT}$ MEMS Switch Contact", IEEE Holm Conference on Electrical Contacts, Albuqurque NM, 2018, pp 356-361pp.

[9] T. Kageyama1, K. Shinozaki, L. Zhang, J. Lu, H. Takaki, S. Lee, " Fabrication of an $\mathrm{Au}-\mathrm{Au} /$ carbon nanotube-composite contacts RF-MEMS switch", Micro and Nano Sytem Letters, 2018, 6:6.

[10] W. Johler, "Basic Investigations for Switching of RF Signals", IEEE Holm conference on Electrical Contacts, 2017, 10.1, pp 229238.

[11] C. Chianrabutra, L. Jiang, A. P. Lewis, and J. W. McBride, "Evaluating the influence of current on the wear processes of $\mathrm{Au} / \mathrm{Cr}-\mathrm{Au} / \mathrm{MWCNT}$ switching surfaces," in 59th IEEE Holm Conference on Electrical Contacts, Newport, RI, 2013, pp. 344349.

[12] K. W. Gilbert, S. Mall, and K. D. Leedy, "Characterization of Gold-Gold Microcontact Behavior Using a Nanoindenter Based Setup," Journal of Adhesion Science and Technology, 2010, vol. 24, pp. 2597-2615.

[13] T. V. Laurvick, and R. A. Coutu, “Improving Gold/Gold Microcontact Performance and Reliability Under Low-Frequency AC Through Circuit Loading," IEEE Transactions on Components, Packaging and Manufacturing Technology, 2017, vol. 7, no. 3, pp. 345-353.

[14] M. Libansky, J. Zima, J. Barek, A. Reznickova, V. Svorcik, and H. Dejmkova, "Basic electrochemical properties of sputtered gold film electrodes,” Electrochimica Acta, 2017, vol. 251, pp. 452460.

[15] J. W. McBride, H. Liu, C. Chianrabutra, and A. P. Lewis, "The Wear of Hot Switching Au/Cr-Au/MWCNT Contact Pairs for MEMS Contacts,” Ieice Transactions on Electronics, 2015, vol. E98c, no. 9, pp. 912-918.
[16] M. P. Down, L. Jiang, and J. W. McBride, "Investigating the benefits of a compliant gold coated multi-walled carbon nanotube contact surface in micro-electro mechanical systems switching," Applied Physics Letters, 2015, vol. 107 (7), 071901.

[17] A. P. Lewis, J. W. McBride, and L. Jiang, "Evolution of Voltage Transients During the Switching of a MEMS Relay With Au/MWCNT Contacts," IEEE Transactions on Components, Packaging and Manufacturing Technology, 2015, vol. 5, no. 12, pp. 1747-1754.

[18] T. G. Bull, and J. W. McBride, "In-Situ Contact Surface Characterization in a MEMS Ohmic Switch under Low Current Switching,” MDPI Technologies, 2018, vol. 2, no. 6, pp. 47.

[19] Taicaan Technologies XYRIS 4000CL, non contact confocal laser metrology system, http://www.taicaan.com

[20] H. Liu, J.W. McBride, “A Finite Element Based Contact Resistance Model for Rough Surfaces: Applied to a Bi-layered Au/MWCNT Composite", IEEE Transactions on Components, Packaging and Manufacturing Technology, 2018, Vol: 8, (6), pp 919 - 926.

[21] P.G Slade (Ed) "Electrical Contacts, Principles and Applications, edition”. Chapter 1.

[22] P.G Collins, M. Hesam, M. Arnold, R Martel, and Ph. Avouris, "Current saturation and electrical breakdown in multiwalled carbon nanotubes”, Physical Review Letters, 2001, Vol 86, No 14, pp3128, 3131.

[23] J.Y. Huang etal, “Atomic-scale imaging of Wall-by-wall breakdown and concurrent transport measurements in multiwall carbon nanotubes”, Physical Review Letters, 2005, PRL, 94, 236802, pp 4.

[24] M. Tsutsui, Y. Taninouchi, S. Kurokawa, A. Sakai, "Electrical breakdown of short multiwalled carbon nanotubes", 2006, J Ap Phy. 100, 094302, pp 5. 